\title{
Analog circuit design and optimal synchronization of a modified Rayleigh system
}

\author{
Michaux Kountchou • Patrick Louodop • \\ Samuel Bowong • Hilaire Fotsin • Saïdou
}

Received: 12 December 2014 / Accepted: 18 February 2016 / Published online: 5 March 2016

(C) Springer Science+Business Media Dordrecht 2016

\begin{abstract}
This paper addresses the problem of optimization of the synchronization of a chaotic modified Rayleigh system. We first introduce a four-dimensional autonomous chaotic system which is obtained by the modification of a two-dimensional Rayleigh system.
\end{abstract}

M. Kountchou $(\varangle) \cdot$ P. Louodop · H. Fotsin

Laboratory of Electronics and Signals Processing,

Department of Physics, Faculty of Science, University of

Dschang, P.O. Box 67, Dschang, Cameroon

e-mail: kountchounoube@yahoo.fr

M. Kountchou · Saïdou

Nuclear Technology Section, Institute of Geological and

Mining Research, P.O. Box 4110, Yaoundé, Cameroon

\section{S. Bowong}

Laboratory of Applied Mathematics, Department of Mathematics and Computer Science, Faculty of Science, University of Douala, P.O. Box 24157, Douala, Cameroon

S. Bowong

UMI 209 IRD\&UPMC UMMISCO, Bondy, France

S. Bowong

LIRIMA, Project Team GRIMCAPE, Yaoundé, Cameroon

\section{S. Bowong}

The African Center of Excellence in Information and Communication Technologies (CETIC), University of Yaounde 1, Yaoundé, Cameroon

Saïdou

Nuclear Physics Laboratory, Faculty of Science, University of Yaounde I, P.O. Box 812, Yaoundé, Cameroon

\section{P. Louodop}

Instituto de Física Teórica, UNESP, Universidade Estadual

Paulista, Rua Dr. Bento Teobaldo Ferraz 271, Bloco II,

Barra Funda, São Paulo 01140-070, Brazil
Some basic dynamical properties and behaviors of this system are investigated. An appropriate electronic circuit (analog simulator) is proposed for the investigation of the dynamical behavior of the proposed system. Correspondences are established between the coefficients of the system model and the components of the electronic circuit. Furthermore, we propose an optimal robust adaptive feedback which accomplishes the synchronization of two modified Rayleigh systems using the controllability functions method. The advantage of the proposed scheme is that it takes into account the energy wasted by feedback coupling and the closed loop performance on synchronization. Also, a finite horizon is explicitly computed such that the chaos synchronization is achieved at an established time. Numerical simulations are presented to verify the effectiveness of the proposed synchronization strategy. Pspice analog circuit implementation of the complete masterslave controller system is also presented to show the feasibility of the proposed scheme.

Keywords Modified Rayleigh system · Chaos synchronization · Optimal feedback control · Controllability function $\cdot$ Pspice analog circuit implementation

\section{Introduction}

In recent years, a great interest has been devoted to theoretical, numerical and experimental investigations to understand the behavior of nonlinear oscillators. 
Theoretical (fundamental) investigations reveal their rich and complex behavior, and the experimental (selfexcited oscillators) describes the evolution of many biological, chemical, physical, mechanical and industrial systems. The interest devoted to chaos by many scientists is due to the fact that this phenomenon appears in various fields, from mathematics, physics, biology and chemistry, to engineering, economics and medicine and elsewhere [1-7]. Consequently, there are many opportunities for application of chaos. For example, Lord Rayleigh came to the problem through his interest in understanding how musical instruments generate sound. In 1883 [8], he introduced an autonomous second-order nonlinear ordinary differential equation to study the oscillations of the violin chords. The dynamics generated by it is close to the dynamics generated by the Van der Pol equation [9]. The Rayleigh equation [8] was introduced in 1979 by Diener in order to study the duck phenomenon [10,11]. Subsequently, it was found that, for certain values of the parameters, the dynamics generated can be very complicated, presenting cascades of bifurcations and chaotic regions.

There are many opportunities for exploitation of chaos: synchronized chaos and mixing with chaos [12], encoding information with chaos [13,14], anti-control of chaos [15], tracking of chaos [16], targeting of chaos [17] and so on. These last years, great efforts have been devoted to controlling and synchronizing chaotic oscillators. Several strategies to control chaos have been proposed and investigated with the objective of stabilizing equilibria or periodic orbits embedded in chaotic attractors [18-21]. The interest in synchronization lies on the potential applications in areas such as biological oscillators, animal gaits and secure communication [13,14]. Moreover, it has been shown that deterministic chaos is not the only source of irregular oscillations since stable periodic systems may also oscillate irregularly when subject to small random noise. Thus, robust synchronization is needed particularly in secure communication systems in order to counteract the effects of external perturbations on the process [14,22-25]. A wide variety of approaches have been proposed for the synchronization of chaotic systems among which are the linear and nonlinear feedback, the time-delay feedback $[26,27]$, the adaptive control [19,23,24,26,28-30], the impulsive control [31], the backstepping control [32-34] and so on. Several studies have also considered the cases where the oscillators models were partially or totally unknown $[18,30,34,35]$. The techniques used generally lead to acceptable performance.

Nevertheless, these methods in general have a drawback, because their physical implementation would appear to be a very hard task. Furthermore, the most common approaches are the numerical simulation associated with the well-known analytical perturbation methods. However, it is well known that with such techniques appear problems related to time integration. Indeed, even with very fast workstations, scanning parameter spaces turns out to be a very slow process. Moreover, to the best of authors' knowledge, there exists no method that can help predict the duration of the transient phase of a numerical simulation. The ana$\log$ simulation offers the way to tackle such difficulties. This is one of the major reasons for the increasing interest devoted to this type of simulation for the analysis of nonlinear and chaotic physical systems [27,36-43]. Indeed, a properly designed circuit can provide sufficiently good real-time results faster than a numerical simulation on a fast computer. Moreover, analog computation reveals itself to be very powerful for a large number of specific problems such as pattern recognition and image processing to just name a few. It seems to be the most natural way to solve many physical problems since many elementary computational primitives are a direct consequence of fundamental laws of physics.

In this paper, we firstly propose a four-dimensional autonomous chaotic system obtained by the modification of a two-dimensional Rayleigh system. The dynamical behaviors of the proposed oscillator are investigated. The bifurcation structures of the system are analyzed. An appropriate electronic circuit (ana$\log$ simulator) is proposed for the investigation of the dynamical behavior of the system. Correspondences are established between the coefficients of the system model and the components of the electronic circuit. A comparison of Pspice and numerical results shows a very good agreement.

Secondly, we use controllability functions method [44] to propose an optimal robust feedback coupling which accomplishes the synchronization between two modified Rayleigh oscillators at a finite time. The expression of the synchronization time is explicitly computed. The advantage of the proposed scheme is that it takes into account the energy wasted by the feedback coupling and the closed loop performance on syn- 
chronization. The robustness of the feedback coupling against model uncertainties is shown through numerical and Pspice simulations.

The structure of the paper is as follows: Sect. 2 deals with the dynamics of the proposed system. The bifurcation structures of the system are investigated numerically. An appropriate electronic circuit is proposed for the investigation of the dynamical behavior of the system. Section 3 investigates the optimal robust synchronization of such proposed systems. Numerical and Pspice simulations are given to show the effectiveness and applicability of the proposed synchronization method. The concluding remarks are given in Sect. 4.

\section{The system and its dynamics}

\subsection{The system}

The autonomous second-order nonlinear ordinary differential equation introduced in 1883 by Lord Rayleigh [8] is given by:

$\frac{\mathrm{d}^{2} x}{\mathrm{~d} t^{2}}+\frac{1}{3}\left(\frac{\mathrm{d} x}{\mathrm{~d} t}\right)^{3}-\frac{\mathrm{d} x}{\mathrm{~d} t}+x=0$,

As it is shown in [38,39], the Rayleigh equation (1) can be converted to a Jerk system as follows:

$\frac{\mathrm{d}^{4} x}{\mathrm{~d} t^{4}}=-\frac{\mathrm{d}^{3} x}{\mathrm{~d} t^{3}}-\frac{\mathrm{d}^{2} x}{\mathrm{~d} t^{2}}-\frac{1}{3}\left(\frac{\mathrm{d} x}{\mathrm{~d} t}\right)^{3}+\frac{\mathrm{d} x}{\mathrm{~d} t}-x$.

The state space representation of this Jerk system yields:

$$
\left\{\begin{array}{l}
\dot{x}_{1}=x_{2}, \\
\dot{x}_{2}=x_{3}, \\
\dot{x}_{3}=x_{4}, \\
\dot{x}_{4}=-x_{4}-x_{3}-\frac{x_{2}^{3}}{3}+x_{2}-x_{1},
\end{array}\right.
$$

where $x_{1}=x, x_{2}=\frac{\mathrm{d} x}{\mathrm{~d} t}, x_{3}=\frac{\mathrm{d}^{2} x}{\mathrm{~d} t^{2}}$, and $x_{4}=\frac{\mathrm{d}^{3} x}{\mathrm{~d} t^{3}}$. To achieve the chaotification for system Eq. (3), three constant parameters $b, c$ and $d$ with two innovation terms $x_{1}$ and $x_{4}$ are introduced as follows:

$$
\left\{\begin{array}{l}
\dot{x}_{1}=x_{2}-b x_{1}+c x_{4}, \\
\dot{x}_{2}=x_{3}, \\
\dot{x}_{3}=d x_{4}, \\
\dot{x}_{4}=-x_{4}-x_{3}-\frac{x_{2}^{3}}{3}+x_{2}-x_{1},
\end{array}\right.
$$

where $b, c$ and $d$ are positive constants.

\subsection{Basic dynamics analysis}

This section analyzes basic dynamic characteristics of the proposed chaotic system (4), including dissipation characteristic, the bifurcation diagram and the maximum Lyapunov exponent spectrum analysis.

\subsubsection{Dissipation and existence of chaotic attractor}

The divergence of the modified four-dimensional system Eq. (4) is

$\nabla V=\frac{\partial \dot{x}_{1}}{\partial x_{1}}+\frac{\partial \dot{x}_{2}}{\partial x_{2}}+\frac{\partial \dot{x}_{3}}{\partial x_{3}}+\frac{\partial \dot{x}_{4}}{\partial x_{4}}=-(1+b)<0$.

Thus, since $\nabla V<0$, one can conclude that system (4) is dissipative; that is, a volume element $V_{0}$ is contracted by the flow into a volume element $V_{0} e^{-(1+b) t}$ in time $t$. This means that each volume containing the trajectory of the dynamical system (4) shrinks to zero as $t \rightarrow \infty$ at an exponential rate $1+b$. Consequently, all the trajectories of system (4) ultimately arrive to an attractor.

\subsubsection{Bifurcation, Lyapunov exponent and chaotic behavior}

According to the chaos theory, the Lyapunov exponents measure the exponential rates of divergence and convergence of nearby trajectories in phase space of system (4). System (4) is solved numerically to define routes to chaos. Here, the types of motion are identified using two indicators. The first indicator is the bifurcation diagram, the second being the largest $1 \mathrm{D}$ numerical Lyapunov exponent denoted by

$\lambda_{\max }=\lim _{t \rightarrow \infty}\left[\frac{1}{t} \ln (\mathrm{d}(t))\right]$,

where

$\mathrm{d}(t)=\sqrt{\left(\delta x_{1}\right)^{2}+\left(\delta x_{2}\right)^{2}+\left(\delta x_{3}\right)^{2}+\left(\delta x_{4}\right)^{2}}$,

and computed from the variational equations obtained by perturbing the solutions of system (4) as follows: $x_{1} \rightarrow x_{1}+\delta x_{1}, x_{2} \rightarrow x_{2}+\delta x_{2}, x_{3} \rightarrow x_{3}+\delta x_{3}$ and $x_{4} \rightarrow x_{4}+\delta x_{4}$. In Eq. (6), $d(t)$ represents the distance between neighboring trajectories. Asymptotically, $\mathrm{d}(t)=e^{\lambda_{\max } t}$. Thus, if $\lambda_{\max }>0$, the neighboring trajectories diverge and the states of the oscillator are chaotic. For $\lambda_{\max }<0$, these trajectories converge and the states of the oscillator are nonchaotic. 
Fig. 1 Bifurcation diagram for the varying parameter $b$ : $\mathbf{a}$ in $[0,1], \mathbf{b}$ in $[1,60]$

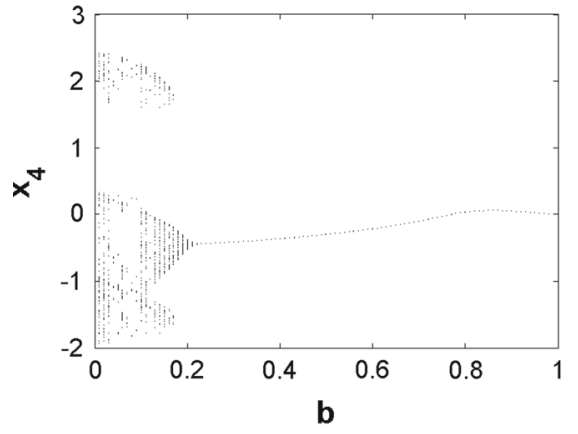

(a)

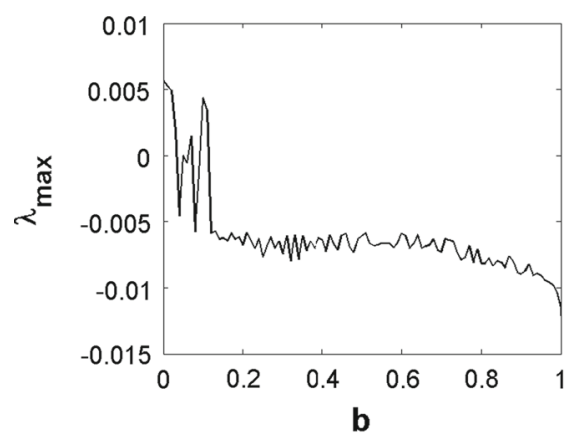

(a)

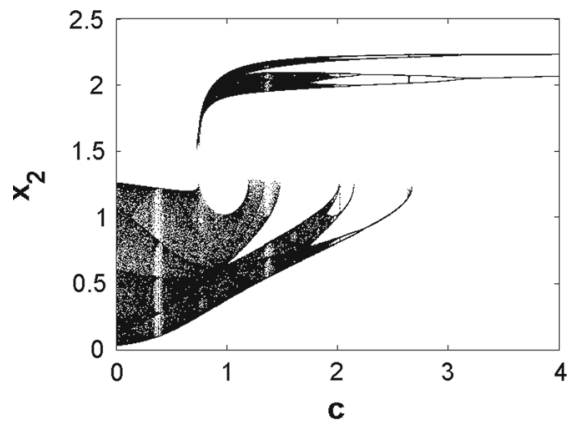

(a)

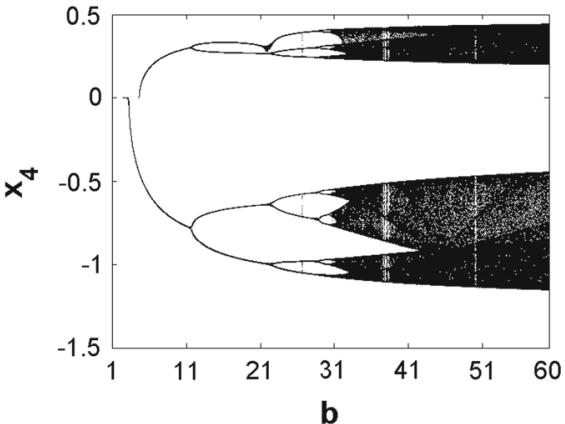

(b)

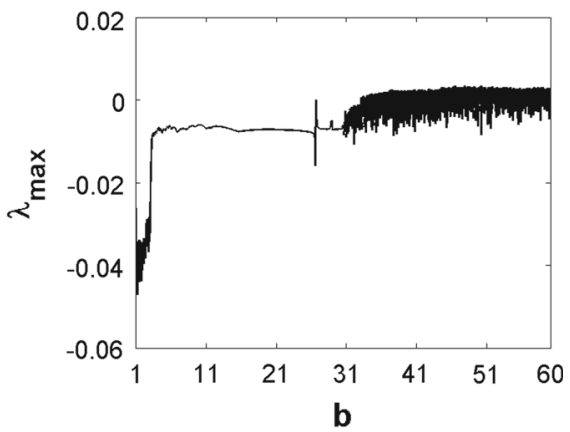

(b)

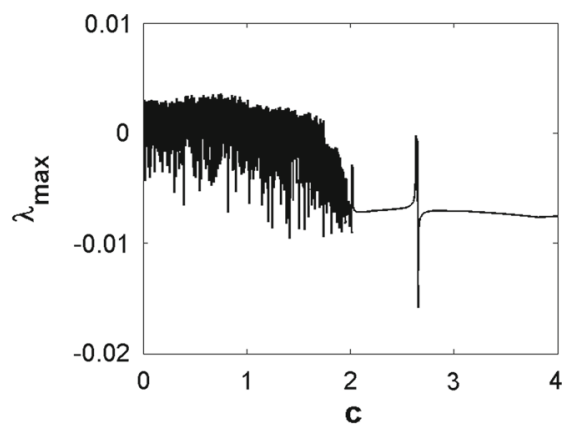

(b)
Finally $\lambda_{\max }=0$ corresponds to torus states of the oscillator. We first consider the case when $c=1.25$, $d=2.5$, and $b$ is varied. Then, the system can generate chaotic dynamical behaviors in a wide region as shown in Figs. 1 and 2, which present the bifurcation diagram and the maximum Lyapunov versus the parameter $b$. Consider now the case when $b=40$, $d=2.5$, and $c$ is varied. Numerical results are depicted in Fig. 3 from which when the control parameter $c$ is varied, a very rich and striking dynamic behavior is observed.

\subsection{Analog circuit implementation}

The analog computer implementation is a convenient tool to scan the parameter range in order to find the proper parameter values for a numerical simulation. Another advantage of such an approach compared to numerical computation is that there is no need to wait for long transient times. This may serve to justify the increasing interest devoted to this type of implementation to the analysis of nonlinear and chaotic systems. In fact, analog simulation can serve as a powerful 
Fig. 4 Analog circuit of a modified Rayleigh system (4)

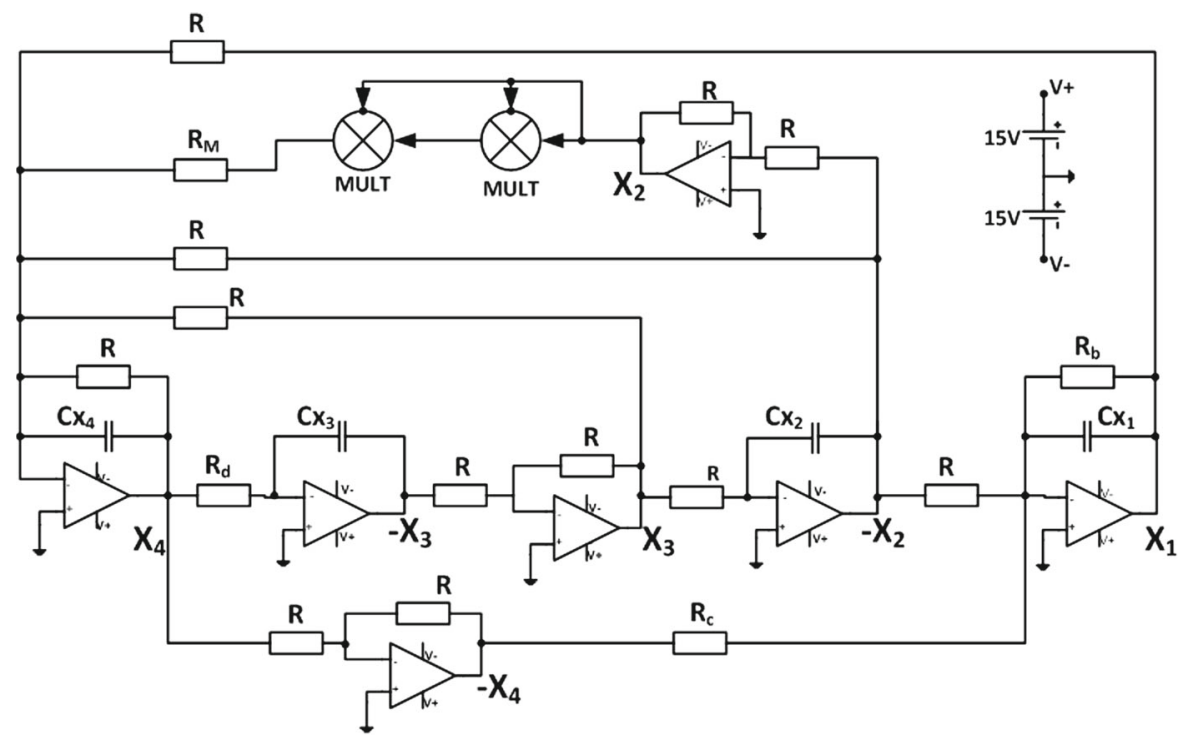

tool to qualitatively describe quickly and cheaply the features of complex dynamics and, therefore, suggest real experiments or serve as guides for more accurate numerical simulations. Then, the goal of this section is to design an appropriate analog simulator for the investigation of the model described by Eq. (4) in order to validate and support theoretical results. Numerical phase portraits are compared to Pspice ones.

The schematic diagram of the complete electronic simulator used to investigate the chaotic behavior of system (4) is shown in Fig. 4. The discrete electronics components such as resistors, capacitors, operational amplifiers ( $T$ L084) are used to construct the circuit. The $T L 084$ are operational amplifiers with Junction Field-Effect Transistors in input (JFETs-input opamps). Each operational amplifier incorporates wellmatched high-voltage JFET and bipolar transistors in the same integrated circuit. The device features are high slew rates, low input bias and offset currents and low offset voltage temperature coefficient. This is significant to reduce sensitivity to circuit parameter values. The electronic multipliers (MULT) are the analog device AD633JN versions of the AD633 four-quadrant voltage multiplier chips. They are used to implement the nonlinear term of the system. Applying the Kirchhoff laws, the circuit of Fig. 4 is described by the following equations:

$$
\left\{\begin{array}{l}
\dot{V}_{C_{x_{1}}}=V_{C_{x_{2}}}-\frac{1}{10^{4} R_{b} C_{x_{1}}} V_{C_{x_{1}}}+\frac{1}{10^{4} R_{c} C_{x_{1}}} V_{C_{x_{4}}}, \\
\dot{V}_{C_{x_{2}}}=V_{C_{x_{3}}}, \\
\dot{V}_{C_{x_{3}}}=\frac{1}{10^{4} R_{d} C_{x_{3}}} V_{C_{x_{4}}}, \\
\dot{V}_{C_{x_{4}}}=-V_{C_{x_{4}}}-V_{C_{x_{3}}}-\frac{1}{10^{4} R_{M} C_{x_{4}}} V_{C_{x_{4}}}^{3}+V_{C_{x_{2}}}-V_{C_{x_{1}}},
\end{array}\right.
$$

where

$$
\begin{aligned}
& b=\frac{1}{10^{4} R_{b} C_{x_{1}}}, \quad c=\frac{1}{10^{4} R_{c} C_{x_{1}}}, \\
& d=\frac{1}{10^{4} R_{d} C_{x_{3}}}, \quad \frac{1}{3}=\frac{1}{10^{4} R_{M} C_{x_{4}}}, \\
& V_{C_{x_{1}}}=x_{1}, \quad V_{C_{x_{2}}}=x_{2}, \quad V_{C_{x_{3}}}=x_{3} \quad \text { and } \\
& V_{C_{x_{4}}}=x_{4} .
\end{aligned}
$$

The time unit is $10^{-4} \mathrm{~s}$. It is worth mentioning that the timescaling process offers the analog devices the possibility to operate under their bandwidth. The timescaling process is also of high importance while performing analog computation. It offers the possibility to simulate the behavior of the system at any given frequency by performing an appropriate timescaling that consists of expressing the MATLAB time variable $T_{\mathrm{M}}$ versus the Pspice computation time variable $T_{\mathrm{S}}$ :

$T_{\mathrm{S}}=\mathrm{RCT}_{\mathrm{M}}=10^{-a} T_{\mathrm{M}}$,

where $a$ is a positive integer depending on the values of the resistors and capacitors used in the analog simulation [37]. 
Fig. 5 a, c Chaotic attractors obtained through numerical simulations for $b=0$ when $c=1.25$ and $d=2.5$. b, $\mathbf{d}$ Chaotic attractors obtained through Pspice simulations for $R_{c}=8 \mathrm{k} \Omega, R_{d}=4 \mathrm{k} \Omega$, and $R_{b} \rightarrow \infty$ (disconnected)

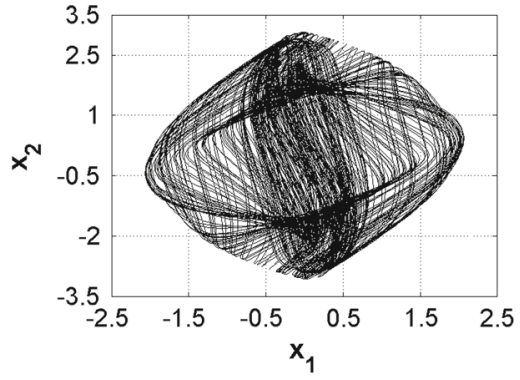

(a)

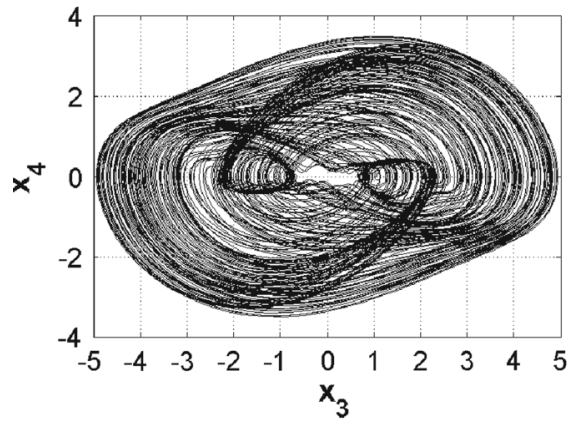

(c)

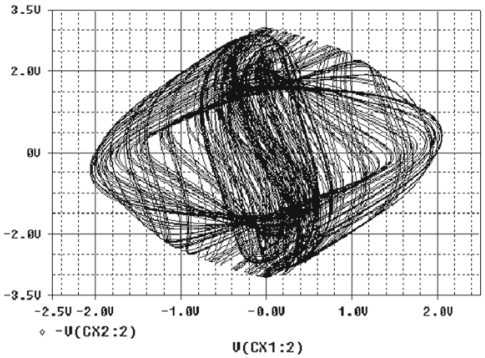

(b)

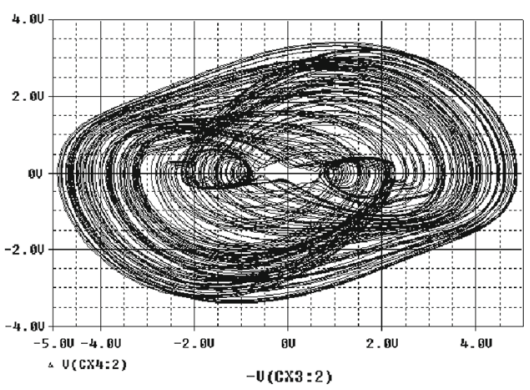

(d)
The following values of circuits components are selected: $C_{x_{1}}=C_{x_{2}}=C_{x_{3}}=C_{x_{4}}=C=10 \mathrm{nF}$, $R=10 \mathrm{k} \Omega, R_{b}=250 \Omega, R_{c}=8 \mathrm{k} \Omega, R_{d}=4 \mathrm{k} \Omega$, $R_{M}=30 \mathrm{k} \Omega$, the voltage source is set at $\pm 15 \mathrm{Vdc}$. The choice of these values is justified by our wish to use the same sets of system parameters for both numerical and experimental studies. It is noted that the effects of varying parameter $b$ on the dynamics of the system can be analyzed by monitoring a single resistor $R_{b}$ while keeping the rest of electronic components values constant.

Figures 5 and 6 show the comparison between the results of numerical and Pspice simulations of the chaotic behavior of the modified Rayleigh system (4). Figures $5 \mathrm{a}-\mathrm{c}$ and $6 \mathrm{a}-\mathrm{c}$ show the results of numerical simulations for $b=0$ and $b=40$ when $c=1.25$ and $d=2.5$, respectively. From these figures, it clearly appears that for the chosen set of parameters, the modified Rayleigh system (4) presents strictly different chaotic attractors. The results of Pspice simulations are shown in Figs. 5b-d and 6b-d when $R_{b}=\rightarrow \infty$ ( $R_{b}$ is disconnected) and $R_{b}=250 \Omega$, respectively. From these figures, it is evident that chaotic attractors obtained through Pspice simulations are very close to the numerically computed results. The experimental results in Fig. 7 also exhibit a good qualitative agree- ment between the experimental realizations and the numerical simulations.

\section{Optimal synchronization}

In this section, we study the synchronization between two identical modified Rayleigh systems. The motivation of such study comes to the fact that the Rayleigh system is an acoustical system which can be used to understand how musical instruments generate sound. This modified system has a very rich and striking dynamic behavior, and it can generate chaotic dynamical behaviors in a wide region as confirmed by the bifurcation diagrams in Figs. 1 and 3. Also, its analysis and physical implementation are particularly straightforward.

\subsection{Synchronization problem}

In this section, we state the synchronization problem. Let us consider the following chaotic system as the drive system:

$\left\{\begin{array}{l}\dot{x}_{1}=x_{2}-b x_{1}+c x_{4}, \\ \dot{x}_{2}=f\left(x_{1}, x_{2}, x_{3}, x_{4}\right), \\ \dot{x}_{3}=d x_{4}, \\ \dot{x}_{4}=-x_{4}-x_{3}-\frac{x_{2}^{3}}{3}+x_{2}-x_{1},\end{array}\right.$ 
Fig. 6 a, c Chaotic attractors obtained through numerical simulations for $b=40$ when $c=1.25$ and $d=2.5$. c, $\mathbf{d}$ Chaotic attractors obtained through Pspice simulations for $R_{c}=8 \mathrm{k} \Omega, R_{d}=4 \mathrm{k} \Omega$, and $R_{b}=250 \mathrm{k} \Omega$

Fig. 7 Experimental results. a Experimental setup in operation. Phase portrait in the plan $\left(x_{3}, x_{4}\right)$ for $R_{c}=8 \mathrm{k} \Omega, R_{d}=4 \mathrm{k} \Omega$. b when $R_{b} \rightarrow \infty$

(disconnected) and $\mathbf{c}$ when $R_{b}=250 \Omega$

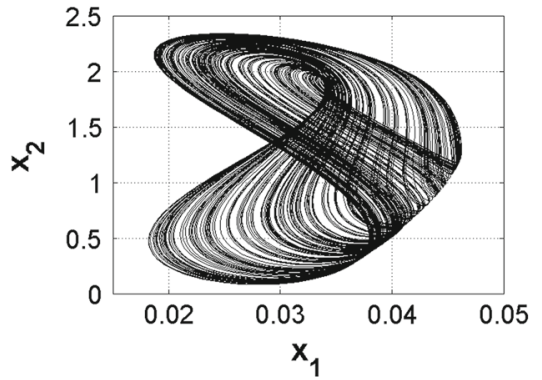

(a)

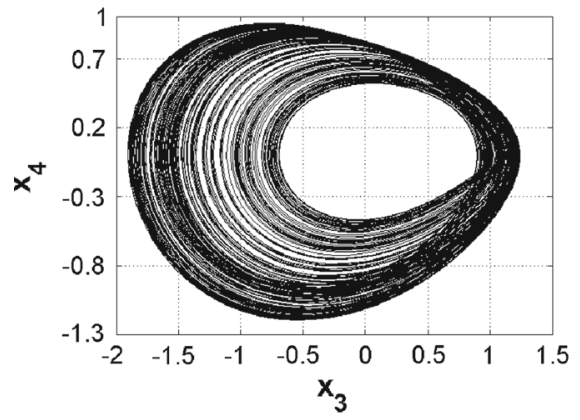

(c)

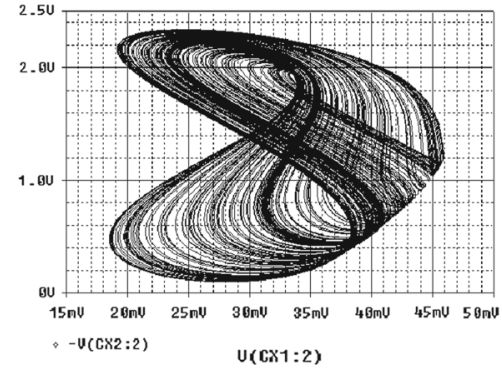

(b)

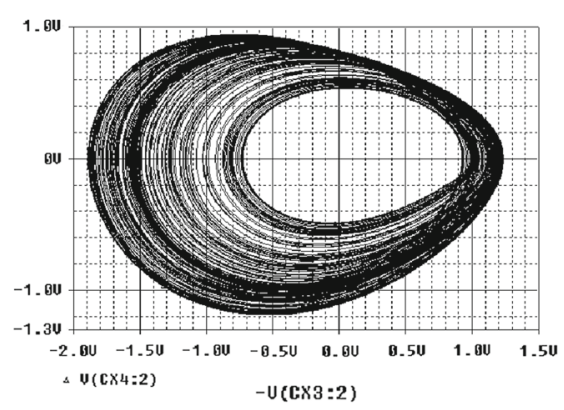

(d)

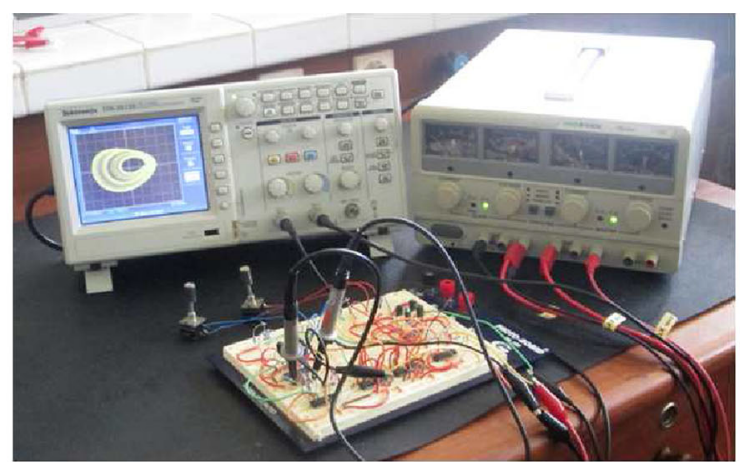

(a)

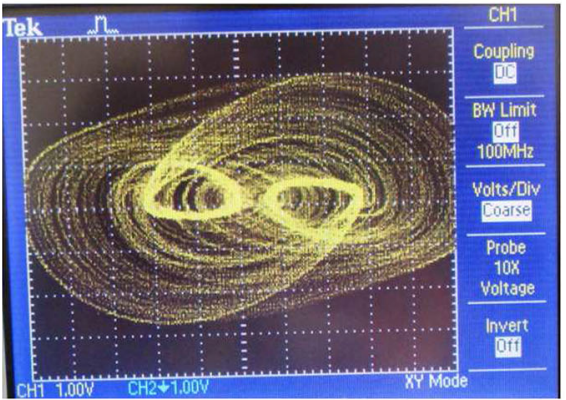

(b)

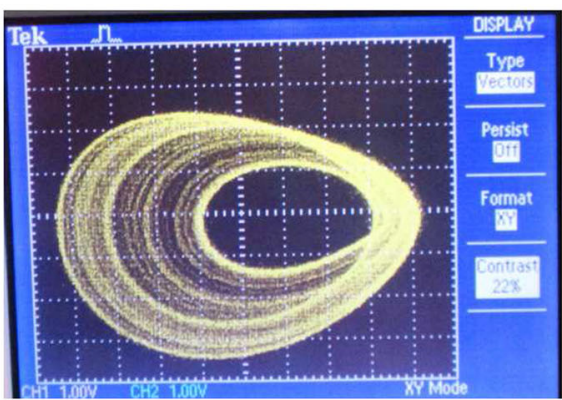

(c) 
where $x=\left(x_{1}, x_{2}, x_{3}, x_{4}\right)^{\mathrm{T}}$ and $f(x)=x_{3}$ is a smooth function.

The slave system is constructed as follows:

$$
\left\{\begin{array}{l}
\dot{y}_{1}=y_{2}-b y_{1}+c y_{4}, \\
\dot{y}_{2}=f\left(y_{1}, y_{2}, y_{3}, y_{4}\right)+u, \\
\dot{y}_{3}=d y_{4}, \\
\dot{y}_{4}=-y_{4}-y_{3}-\frac{y_{2}^{3}}{3}+y_{2}-y_{1},
\end{array}\right.
$$

where $y=\left(y_{1}, y_{2}, y_{3}, y_{4}\right)^{\mathrm{T}}, f(y)=y_{3}$ is a smooth function, and $u$ is the feedback coupling to be chosen. $x_{2}$ and least prior information about the structure of system...'.The synchronization problem can be stated as follows: Given the transmitted signal $x_{2}$ and least prior information about the structure of system (10), to design a feedback coupling $u$ such that which synchronizes the orbits of both the drive and response systems at an established finite time $T$, i.e.,

$\lim _{t \rightarrow T} y_{2}(t) \approx x_{2}(t)$.

Now, let us define the synchronization error as follows:

$e_{i}=y_{i}-x_{i}, \quad i=1,2,3,4$.

Then, the synchronization error dynamics is

$$
\left\{\begin{array}{l}
\dot{e}_{1}=e_{2}-b e_{1}+c e_{4}, \\
\dot{e}_{2}=\Delta F+u \\
\dot{e}_{3}=d e_{4} \\
\dot{e}_{4}=-e_{4}-e_{3}-\frac{1}{3}\left(y_{2}^{3}-x_{2}^{3}\right)+e_{2}-e_{1},
\end{array}\right.
$$

where $\Delta F=f(y)-f(x)=e_{3}$ is a smooth vector field. In this way, the synchronization problem can be seen as the stabilization of Eq. (14) at the origin in a finite horizon. In other words, the problem is to find a robust feedback coupling $u$ such that $\lim _{t \rightarrow T}\|e(t)\| \approx$ 0 (which implies that $x_{i}(t) \approx y_{i}(t)$ for all $t \geq T>0$, $i=1, \ldots, 4)$.

Now, let $\zeta_{1}=e_{1}, \zeta_{2}=e_{3}, \zeta_{3}=e_{4}$. Then, system (14) can be changed into a canonical form [45-49] as follows:

$$
\left\{\begin{array}{l}
\dot{e}_{2}=\Delta F+u \\
\dot{\zeta}_{1}=e_{2}-b \zeta_{1}+c \zeta_{3} \\
\dot{\zeta}_{2}=d \zeta_{3} \\
\dot{\zeta}_{3}=-\zeta_{3}-\zeta_{2}-\frac{1}{3} e_{2}^{3}+e_{2}-\zeta_{1}-G
\end{array}\right.
$$

where

$\Delta F=\zeta_{2}$ and $G=\frac{1}{3}\left(3 x_{2} e_{2}^{2}+3 x_{2}^{2} e_{2}\right)$.
Let $\Delta F=\eta$. Then, following [45-49], system (15) can be rewritten in the following extended form:

$$
\left\{\begin{array}{l}
\dot{e}_{2}=\eta+u, \\
\dot{\eta}=\Gamma\left(e_{2}, \eta, \zeta, u, \dot{u}\right) \\
\dot{\zeta}=\Psi\left(e_{2}, \zeta\right)
\end{array}\right.
$$

where

$$
\begin{aligned}
& \zeta=\left(\zeta_{1}, \zeta_{2}, \zeta_{3}\right)^{\top}, \quad \Gamma\left(e_{2}, \eta, \zeta, u, \dot{u}\right)=\dot{\zeta}_{2}=d \zeta_{3} . \\
& \Psi\left(e_{2}, \zeta\right)=\left(e_{2}-b \zeta_{1}+c \zeta_{3}, d \zeta_{3},-\zeta_{3}-\zeta_{2}\right. \\
& \left.\quad-\frac{1}{3} e_{2}^{3}+e_{2}-\zeta_{1}-G\right)^{\top}
\end{aligned}
$$

At this stage, we point out that system (16) is minimum phase, that is, the zero dynamics $\dot{\zeta}=\Psi(0, \zeta)$ converges to the origin. In other words, the closed loop system is internally stable [41].

To illustrate that system (16) satisfies the minimumphase property, one may prove that $\dot{\zeta}_{1}=e_{2}-b \zeta_{1}+c \zeta_{3}$, $\dot{\zeta}_{2}=d \zeta_{3}$, and $\dot{\zeta}_{3}=-\zeta_{3}-\zeta_{2}-\frac{1}{3} e_{2}^{3}+e_{2}-\zeta_{1}-G$ converge asymptotically to zero when $e_{2}=0$. Note that $\zeta=\left(\zeta_{1}, \zeta_{2}, \zeta_{3}\right)^{\top}$ is bounded. Thus, the zero dynamics can be written as

$\dot{\zeta}=E \zeta$,

where

$E=\left[\begin{array}{ccc}-b & 0 & c \\ 0 & 0 & d \\ -1 & -1 & -1\end{array}\right]$.

For $b=40, c=1.25$ and $d=2.5$, the eigenvalues are $\{-39.96797378,-0.5160131082 \pm$ $1.495237010 i\}$, which is Hurwitz. Thus, for these suitably selected parameters, the zero dynamics subsystem $\dot{\zeta}=E \zeta$ is asymptotically stable. Hence, system (16) is minimum phase. Then, when we have taken actions to achieve $\lim _{t \rightarrow T} e_{2}(t)=0$, the part $\Psi\left(e_{2}, \zeta\right) \rightarrow \Psi(0, \zeta) \rightarrow 0$ as $t \rightarrow \infty$ asymptotically for the so-called minimum-phase character.

\subsection{Design of the feedback coupling}

In this section, the problem of designing $u$ is addressed in such a manner that energy wasted by the feedback is accounted. Indeed, in seeking the optimization of the duration time in the chaos synchronization, the energy demanded to synchronize by a (robust or optimized) feedback can be larger than the available energy by the physical actuator. Therefore, a robust feedback 
coupling that takes into account the behavior of transient response and the feedback coupling effort (i.e., the energy wasted by the feedback coupling action) is necessary and important. The approach developed considers incomplete state measurements and no detailed model of the systems to guarantee robust stability (in fact, robust synchronization). This approach includes an uncertainty estimator and leads to a robust adaptive feedback control scheme. Toward the optimization problem, the first step in our approach is to consider the transitive of states. To this end, the following mixed cost function is defined by quantifying the transient trajectory of the synchronization error [44]:

$J(u)=k^{2} T+\int_{t_{0}}^{T}\left[\left(W e_{2}, e_{2}\right)+(U u, u)\right] \mathrm{d} t, \quad k>0$,

where $t_{0} \geq 0$ is the time at which the synchronization starts and $T>t_{0}$ is the time for which the synchronization error system (14) achieves the desired trajectory $(e=0) ; W \geq 0$ and $U>0$ are the suitable positive constants.

The optimal synchronization problem now consists in finding a control law $u$ such that the master (10) and slave (11) systems synchronize at an established finite time $T$ meanwhile minimizing the mixed cost function (17) (we refer the reader to [44] for the choice of the mixed cost function (17)).

The robust feedback coupling is designed as follows

$$
\begin{gathered}
u\left(e_{2}\right)=-\eta-U^{-1} N^{-1}\left(\theta\left(e_{2}\right)\right) e_{2}, \\
\text { with } \theta\left(e_{2}\right)=T-t
\end{gathered}
$$

$N(t) \in \mathbb{R}$ be a positive function, and solution of the differential Riccati equation:

$\dot{N}=U^{-1}-W N^{2}$.

We have the following results:

Let us designate, for $T>0$ and $e_{2} \in \mathbb{R}$, that

$$
\begin{aligned}
v\left(T, e_{2}\right) & =k^{2} T+\left(N^{-1}(T)\right) e_{2}^{2} \\
v\left(T, e_{2}\right) & =v_{T}^{\prime}\left(T, e_{2}\right), \\
& =k^{2}-\left(U^{-1} N^{-2}(T)-W\right) e_{2}^{2} .
\end{aligned}
$$

Remark 1 At each $e_{2} \in \mathbb{R} \backslash\{0\}$, the function $v\left(T, e_{2}\right)$ achieves its minimum.

In fact, the function $v\left(T, e_{2}\right)$ is continuous (moreover, this function is analytic on $(0, \infty) \times \mathbb{R})$. Since $\|N(T)\|^{-1}\left\|e_{2}\right\|<\left(N^{-1}(T)\right) e_{2}^{2}$ and $\lim _{T \rightarrow+0}\|N(T)\|$
$=0$, it follows that $\lim _{\theta \rightarrow+0} v\left(\theta, e_{2}\right)=+\infty$. On the other hand, it is also clear that $\lim _{T \rightarrow+\infty} v\left(T, e_{2}\right)=$ $+\infty$, so the statement is verified.

We refer to the function that is defined for $e_{2} \neq 0$ by equality of Eq. (18) and defined for $e_{2}=0$ by Eq. (18) with $\theta(0)=0$ as the controllability function [44]. At each $e_{2} \neq 0$, the value of this function coincides with the minimal positive root of the equation $v\left(\theta, e_{2}\right)=0$, at which the function $v\left(\theta, e_{2}\right)$ attains its global minimum (see [44]). Then, the synchronization time $T$ is given by expression of $\theta\left(e_{2}(0)\right)$ which is obtained by the simple resolution of equation $v\left(\theta, e_{2}\right)=0$.

Following [44], some interesting properties of the controllability function $\theta\left(e_{2}\right)$ are given as follows:

Property 1 From $\theta\left(e_{2}\right) \rightarrow 0$, it follows that $e_{2} \rightarrow 0$.

In fact,

$$
\begin{aligned}
\left\|N\left(\theta\left(e_{2}\right)\right)\right\|^{-1}\left\|e_{2}\right\|^{2} & \leq v\left(\theta\left(e_{2}\right), e_{2}\right) \\
& =k^{2} \theta+\left(N^{-1}\left(\theta\left(e_{2}\right)\right) e_{2}^{2}\right) .
\end{aligned}
$$

On the other hand,

$$
\begin{aligned}
v\left(\theta, e_{2}\right) \leq v\left(1, e_{2}\right) & =k^{2}+\left(N^{-1}(1) e_{2}^{2}\right) \\
& \leq k^{2}+\left\|N^{-1}(1)\right\|\left\|e_{2}\right\|^{2} .
\end{aligned}
$$

From Eqs. (22) and (23), we obtain that

$\left\|e_{2}\right\|^{2}\left(\left\|N\left(\theta\left(e_{2}\right)\right)\right\|^{-1}-\left\|N^{-1}(1)\right\|\right) \leq k^{2}$.

Let $\epsilon$ be any positive number such that

$1-\left\|N^{-1}(1)\right\| \epsilon>0$.

Choose $r>0$ such that, if $0<\theta\left(e_{2}\right)<r$, then $\left\|N\left(\theta\left(e_{2}\right)\right)\right\|<\epsilon$; that is, $\left\|N\left(\theta\left(e_{2}\right)\right)\right\|^{-1}>1 / \epsilon$. Then, when $0<\theta\left(e_{2}\right)<r$, because of Eqs. (24) and (25),

$\left\|e_{2}\right\|^{2}\left(1 / \epsilon-\left\|N^{-1}(1)\right\|\right) \leq k^{2}$,

$\left\|e_{2}\right\|^{2} \leq \epsilon k^{2} /\left(1-\left\|N^{-1}(1)\right\| \epsilon\right)$

are fulfilled; hence, Property 1 is obtained.

Property 2 When $\theta\left(e_{2}\right) \rightarrow 0$, then $v\left(\theta\left(e_{2}\right), e_{2}\right) \rightarrow 0$.

Let $\epsilon$ be any positive number and choose $\epsilon>r>0$ so small that when $\theta\left(e_{2}\right)<r,\left(N^{-1}(\epsilon) e_{2}^{2}\right)<\epsilon$ (this is possible because of first statement). Then $v\left(\theta\left(e_{2}\right), e_{2}\right) \leq \nu\left(\epsilon, e_{2}\right)=\left(k^{2}+1\right) \epsilon$, which proves the second statement.

Property 3 For any initial condition $e_{2}(0) \in \mathbb{R}$, $\dot{\theta}\left(e_{2}(t)\right)=-1$ for every $t \in\left[0, \theta\left(e_{2}(0)\right)\right]$ (see [44]). 
Fig. 8 Time evolution of the synchronization errors using numerical simulations. a $e_{2}=y_{2}-x_{2}$; $\mathbf{b} e_{1}=y_{1}-x_{1}$, $e_{3}=y_{3}-x_{3}$, and $e_{4}=y_{4}-x_{4}$

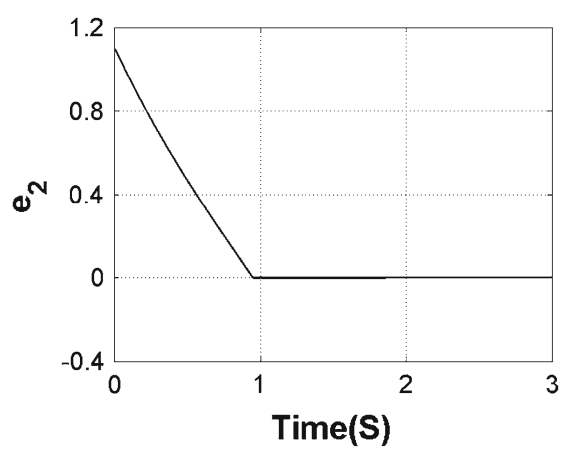

(a)

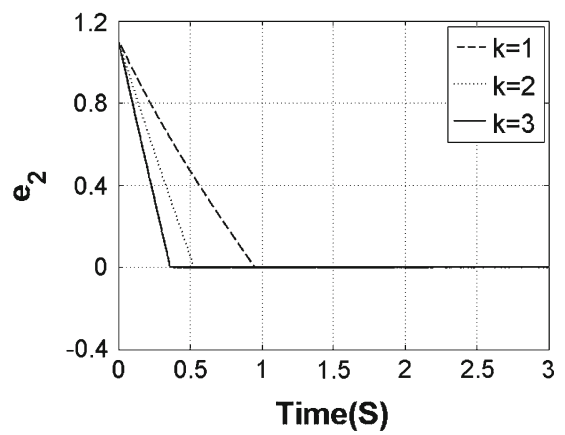

(a)

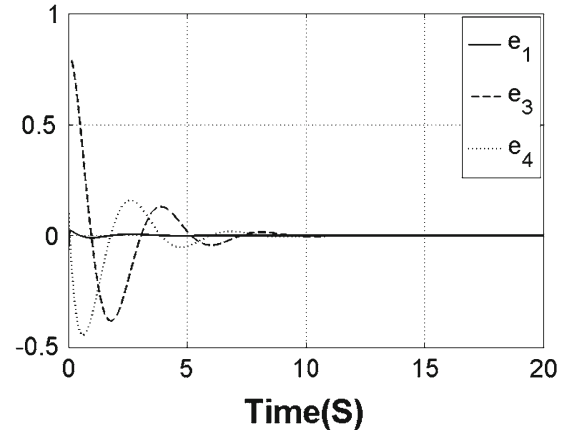

(b)

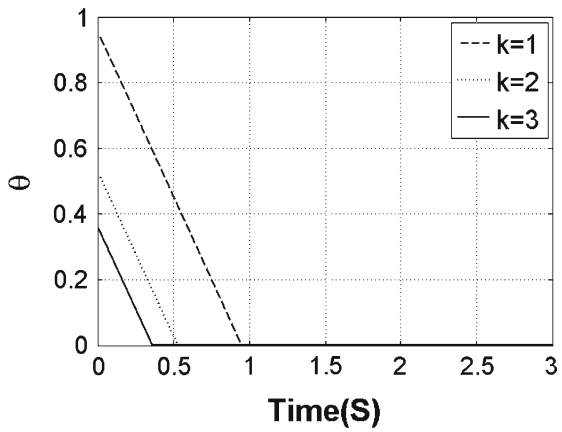

(b)

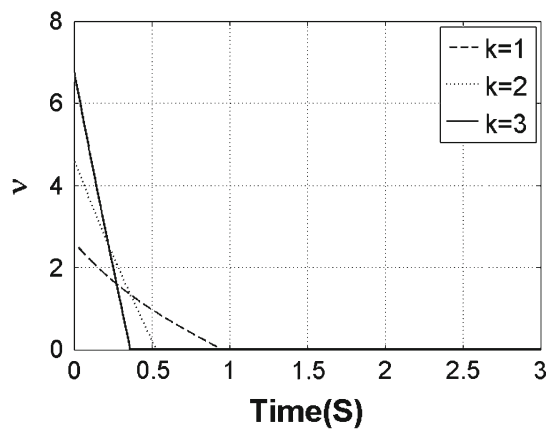

(c)

Proposition 1 Let $\theta\left(e_{2}\right)$ be the controllability function; under the feedback coupling (18), the synchronization error $e_{2}(t)$ converges asymptotically to zero at an established finite time $T=\theta\left(e_{2}(0)\right)$. Moreover, the closed loop performance has a value of the functional (17) $J\left(e_{2}, u\right)=k^{2} \theta\left(e_{2}(0)\right)+$ $N^{-1} \theta\left(e_{2}(0)\right) e_{2}^{2}(0)$.

Proof Since we have $\dot{\theta}\left(e_{2}(t)\right)=-1$, the fact that $\lim _{t \rightarrow \theta\left(e_{2}(0)\right)} e_{2}(t) \rightarrow 0$ follows from

$\lim _{t \rightarrow \theta\left(e_{2}(0)\right)} \theta\left(e_{2}\right)=\lim _{t \rightarrow \theta\left(e_{2}(0)\right)}\left(\theta\left(e_{2}(0)\right)-t\right)=0$.
Let $\epsilon$ be any positive number. Consider the value of the cost functional (17) at the control $u\left(e_{2}\right)$ and the solution $e_{2}(t)$ corresponding to it, and we have that [44]:

$$
\begin{aligned}
J\left(u\left(e_{2}\right)\right)= & \lim _{\epsilon \rightarrow+0} \int_{t_{0}}^{\theta\left(e_{2}(0)\right)-\epsilon}\left[\left(U u\left(e_{2}(t)\right), u\left(e_{2}(t)\right)\right)\right. \\
& \left.+\left(W e_{2}(t), e_{2}(t)\right)\right] d t+k^{2} \theta\left(e_{2}(0)\right), \\
= & \lim _{\epsilon \rightarrow+0} \int_{t_{0}}^{\theta\left(e_{2}(0)\right)-\epsilon}-\dot{v}\left(\theta\left(e_{2}(t)\right), e_{2}(t)\right) d t, \\
= & -\lim _{\epsilon \rightarrow+0} v\left(\theta\left(e_{2}\left(\theta\left(e_{2}(0)\right)-\epsilon\right)\right),\right. \\
& \left.e_{2}\left(\theta\left(e_{2}(0)\right)-\epsilon\right)\right)+v\left(\theta\left(e_{2}(0)\right), e_{2}(0)\right), \\
& \quad \text { with } t_{0}=0
\end{aligned}
$$


Fig. 10 Time evolution of the synchronization errors using numerical simulations, with $2 \%$ of parameter mismatch between the master and slave systems. a $e_{2}=y_{2}-x_{2} ; \mathbf{b}$ $e_{1}=y_{1}-x_{1}, e_{3}=y_{3}-x_{3}$, and $e_{4}=y_{4}-x_{4}$

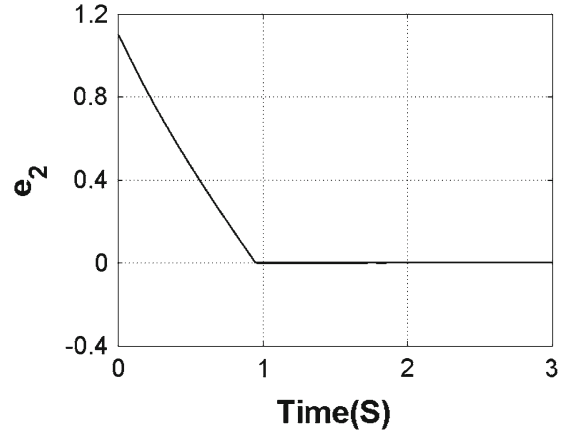

(a)

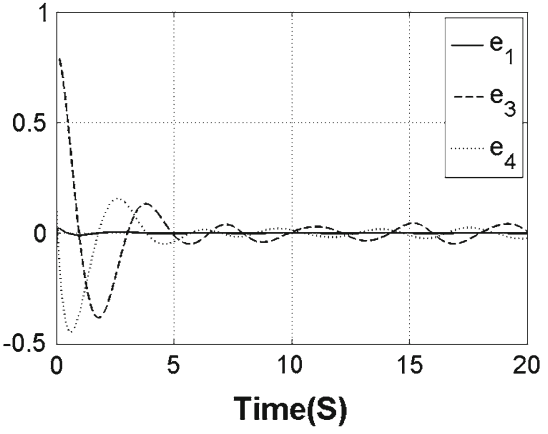

(b)

Fig. 11 Circuit diagram of the drive system (10)

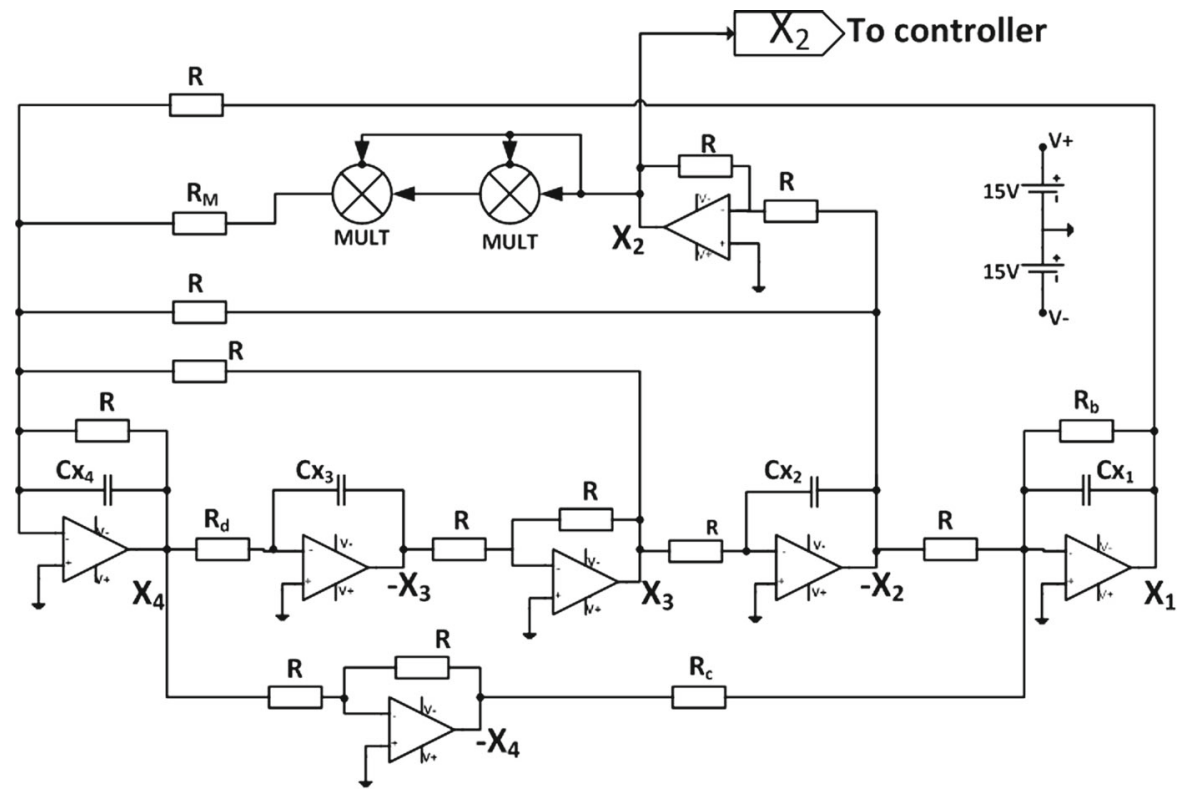

$$
\begin{aligned}
& =v\left(\theta\left(e_{2}(0)\right), e_{2}(0)\right), \\
& =k^{2} \theta\left(e_{2}(0)\right)+N^{-1} \theta\left(e_{2}(0)\right) e_{2}^{2}(0), \\
& =\min _{\theta>0}\left[k^{2} \theta+N^{-1}(\theta) e_{2}^{2}(0)\right] .
\end{aligned}
$$

This completes the proof.

Nevertheless, the linearizing-like feedback (18) is not physically realizable because it requires measurements of the state $e_{2}$ and a perfect knowledge of the nonlinear term $\eta$. Because the linearizing-like feedback (18) must be modified in such a way as to encompass consideration of modeling errors and parameter perturbations. We therefore use the estimation of $e_{2}$ and $\eta$ in such a way that the main characteristics of the linearizing-like feedback (18) are retained. An important advantage of system (16) is that the dynamics of the state $e_{2}$ and the uncertain state $\eta$ can be reconstructed from the output $y=e_{2}$.

As it has been established in $[43,47]$, the problem of estimating $\eta$ can be addressed using a high-gain observer. Thus, the dynamics of the state $\eta$ can be reconstructed from measurements of the output $e_{2}$ in the following way:

$$
\left\{\begin{array}{l}
\dot{\hat{e}}_{2}=\hat{\eta}+u+2 L\left(e_{2}-\hat{e}_{2}\right) \\
\dot{\hat{\eta}}=L^{2}\left(e_{2}-\hat{e}_{2}\right) .
\end{array}\right.
$$

where $\left(\hat{e}_{2}, \hat{\eta}\right)$ are estimated values of $\left(e_{2}, \eta\right)$ and $L>0$ is the so-called high-gain parameter. It has been proved that there exists a sufficiently large value of the highgain parameter $L>L^{*}$, and the dynamics of the estimation error converges exponentially to zero. In addition, the closed loop is stable [47]. 
Fig. 12 Circuit diagram of the response system (11)

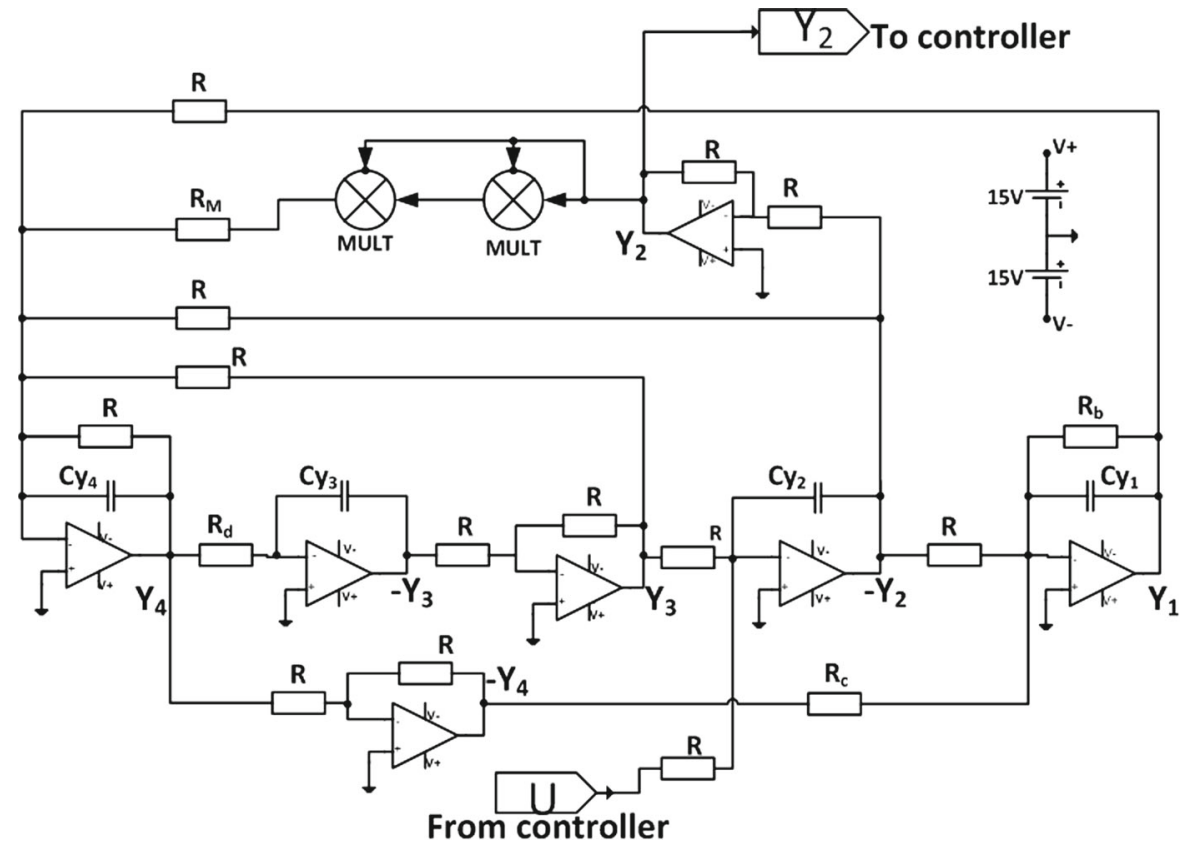

By using the estimated values $\left(\hat{e}_{2}, \hat{\eta}\right)$, the feedback coupling (18) can be written as

$u\left(\hat{e}_{2}\right)=-\hat{\eta}-U^{-1} N^{-1}\left(\theta\left(\hat{e}_{2}\right)\right) \hat{e}_{2}$,

\subsection{Numerical simulations}

In this section, we present the results of numerical simulations and implementation to illustrate and validate the analytical results obtained in the previous section. The parameter values of the drive and response systems are chosen as $b=40, c=1.25, d=2.5$. The initial conditions of the master and slave systems were chosen to be $\left(x_{1}(0), x_{2}(0), x_{3}(0), x_{4}(0)\right)=$ $(0.1,0.9,0.003,0.001), \quad$ and $\quad\left(y_{1}(0), y_{2}(0), y_{3}(0)\right.$, $\left.y_{4}(0)\right)=(0.01,2,0.8,0.1)$. We choose $\left(\hat{e}_{2}(0), \hat{\eta}(0)\right)$ $=(1.1,0)$. The high-gain parameter value was chosen as $L=200$. Considering that $W=1$ and $U=1$, a computation of Riccati equation (19) yields

$N(t)=\frac{e^{2 t}-1}{e^{2 t}+1}$.

The functions $v$ and $v$ are given by

$$
\begin{aligned}
& v\left(\theta, \hat{e}_{2}\right)=k^{2} \theta+\frac{e^{2 \theta}+1}{e^{2 \theta}-1} \hat{e}_{2}^{2}, \\
& v\left(\theta, \hat{e}_{2}\right)=k^{2}-\frac{4 e^{2 \theta}}{\left(e^{2 \theta}-1\right)^{2}} \hat{e}_{2}^{2} .
\end{aligned}
$$

Solving equation $v\left(\theta, \hat{e}_{2}\right)=0$ for $\theta$, we obtain that

$\theta\left(\hat{e}_{2}\right)=\frac{1}{2} \ln \frac{2 \hat{e}_{2}^{2}+k^{2}+2 \sqrt{\hat{e}_{2}^{4}+\hat{e}_{2}^{2} k^{2}}}{k^{2}}$

Note that the other root satisfies $\left(2 \hat{e}_{2}^{2}+k^{2}-2 \sqrt{\hat{e}_{2}^{4}+\hat{e}_{2}^{2} k^{2}}\right) / k^{2}<1$ and so discarded. Thus, the optimal control is given by the formula

$$
\begin{aligned}
u\left(\hat{e}_{2}\right) & =-\hat{\eta}-\frac{e^{2 \theta}+1}{e^{2 \theta}-1} \hat{e}_{2} \\
& =-\hat{\eta}-\frac{\hat{e}_{2}^{2}+k^{2}+\sqrt{\hat{e}_{2}^{4}+\hat{e}_{2}^{2} k^{2}}}{\hat{e}_{2}^{2}+\sqrt{\hat{e}_{2}^{4}+\hat{e}_{2}^{2} k^{2}}} \hat{e}_{2} .
\end{aligned}
$$

For $k=1$ and $\hat{e}_{2}(0)=1.1$, the finite horizon is established at $T=\theta\left(\hat{e}_{2}(0)\right)=0.950346$ s (i.e., the convergence should be attained at time $t \equiv T$ ).

Figure 8 shows the time evolution of the synchronization error. From this figure, one can observe that the synchronization error is stabilized at the origin by the output feedback coupling (29), (34). From Fig. 8a, it clearly appears that a fairly good convergence of $e_{2}$ is obtained in about $0.950346 \mathrm{~s}$ which corresponds to the finite horizon. Note that although the feedback coupling is acting only on the state $e_{2}$, the synchronization errors $e_{1}, e_{3}$ and $e_{4}$ are also stabilized at the origin (minimum-phase character) as it is shown in Fig. 8b. 


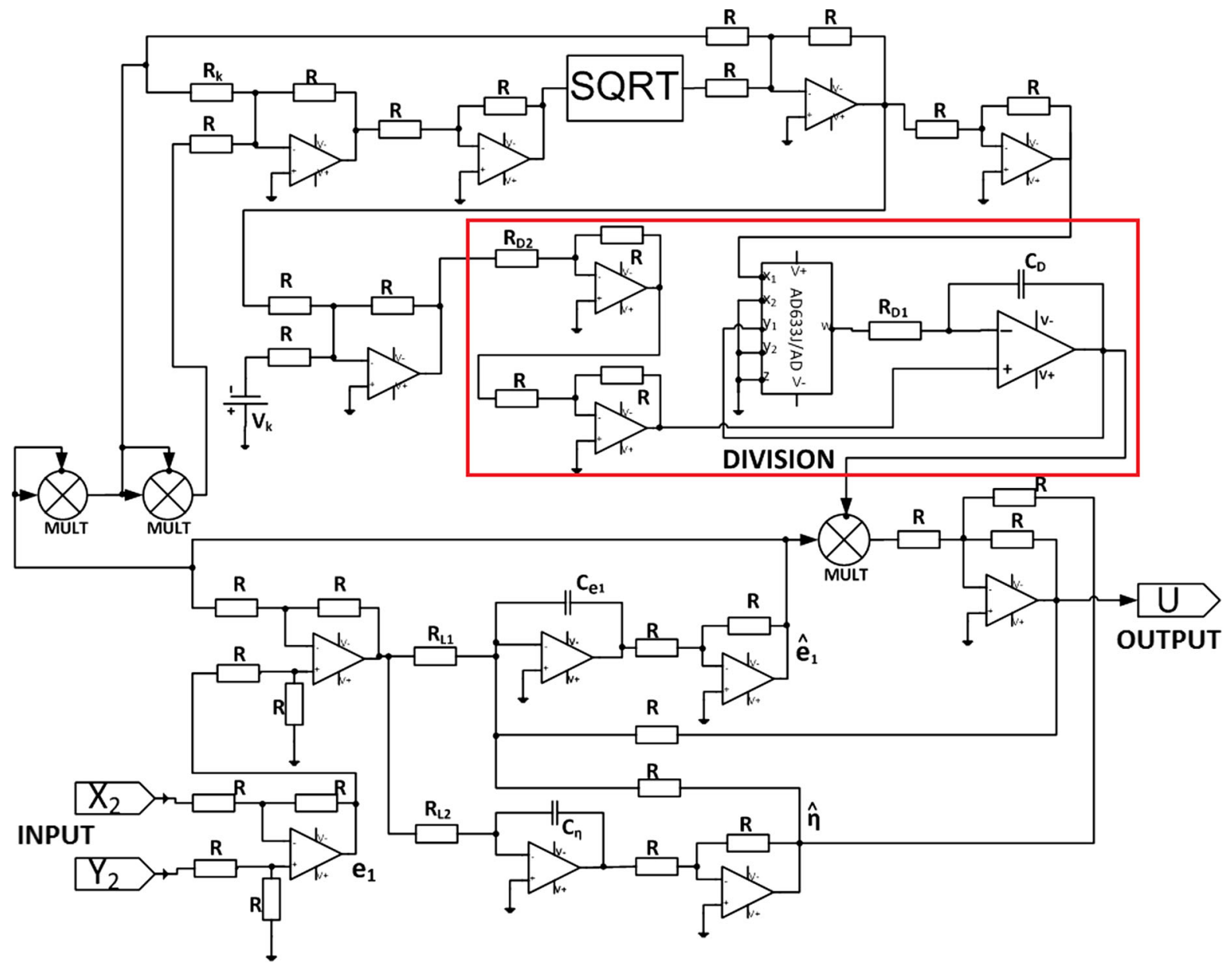

Fig. 13 Circuit diagram of the feedback coupling (29) and (34)

In comparison, one more simulation study is conducted to further verify the effectiveness of the robust feedback controller Eqs. (29), (34). Figure 9 shows the time evolution of the state $e_{2}$, controllability function $\theta\left(\hat{e}_{2}\right)$ and function $v\left(\theta, \hat{e}_{2}\right)$ for three different values of gain $k$ when $\hat{e}_{2}(0)=1.1$; these results confirm the properties 1 and 2 . As predicted by the analysis of formula of $\theta\left(\hat{e}_{2}(0)\right)$ from Eq. (33), the finite horizon is determined by the control gains $k$ and initial condition $\hat{e}_{2}(0)$. Then, it is found that for $\hat{e}_{2}(0)=1.1$ fixed, and for tree values of $k=1, k=2, k=3$, the convergence of $e_{2}$ is obtained, respectively, in about $0.950346,0.525480,0.358911 \mathrm{~s}$ which correspond to the finite horizon determined by $\theta\left(\hat{e}_{2}(0)\right)$ (see Fig. 9).

Now, we investigate the robustness of the proposed scheme with respect to parameter mismatching. The parameter values of the master system (10) are chosen as $b=40, c=1.25, d=2.5$, while the parame- ter values of the slave system (11) were chosen to be $b=39.2, c=1.225, d=2.45$ corresponding to $2 \%$ of parameter mismatches. Figure 10 presents the time evolution of the synchronization errors. From this figure, it is evident that a fairly good convergence of $e_{2}$ is obtained in about the same previous synchronization time $0.950346 \mathrm{~s}$ (see Fig. 8) which corresponds to the finite horizon in spite of the fact that both master and slave systems have different parameters values and different initial states.

\subsection{Pspice implementation}

The aim of this section is to implement a practical setup for the synchronization strategy presented above and to perform Pspice simulation to verify the practical feasibility of the proposed strategy. Using the values of the parameters obtained above, we determine 
Fig. 14 Time evolution of the synchronization error $e_{2}=y_{2}-x_{2}$ using Pspice simulations

Fig. 15 Time evolution of the synchronization errors $e_{1}=y_{1}-x_{1}, e_{3}=y_{3}-x_{3}$, and $e_{4}=y_{4}-x_{4}$ using Pspice simulations
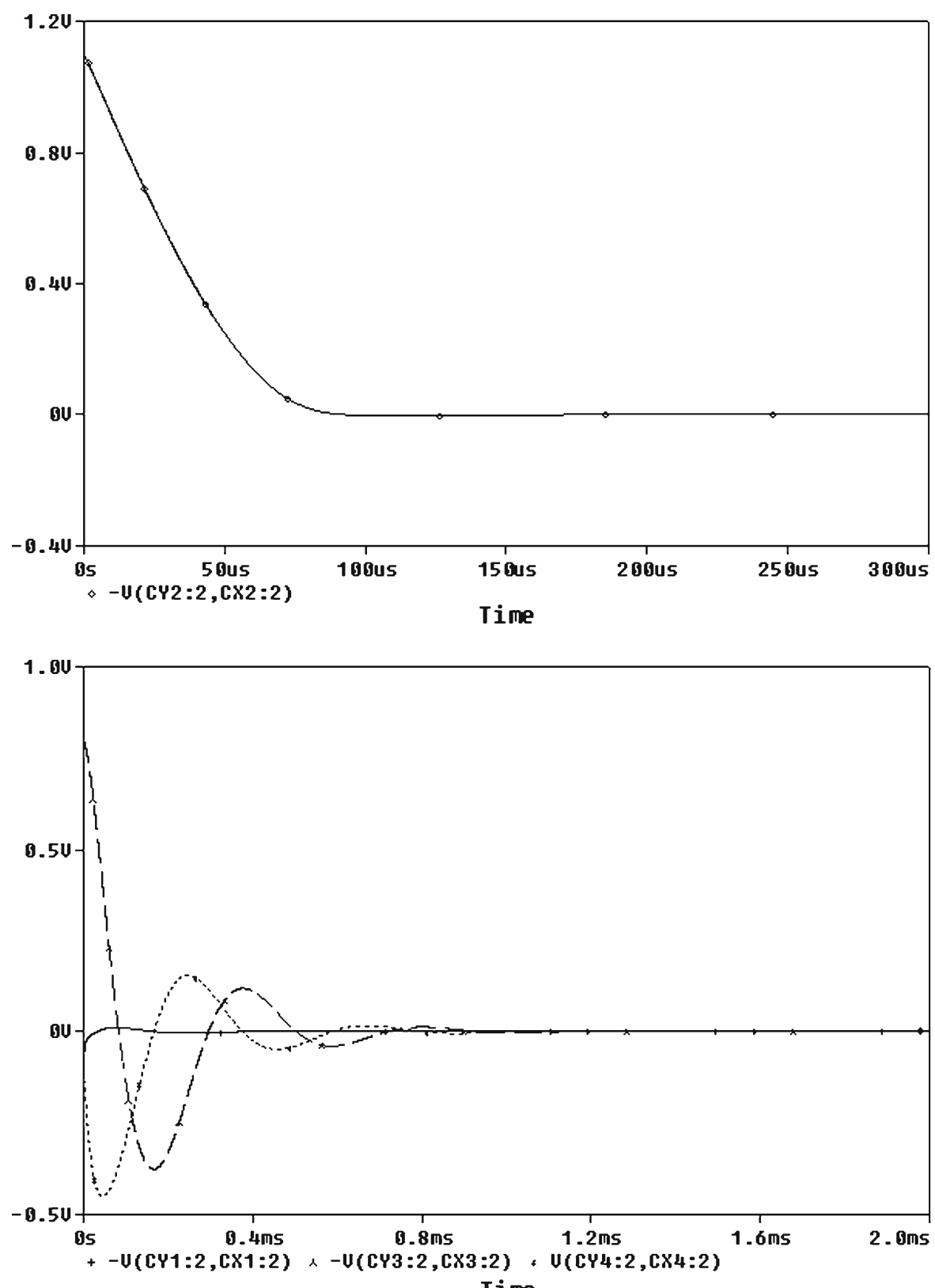

the corresponding electronic coefficients to design and implement the electronic circuit of the synchronization scheme. The values of the parameters of the feedback coupling can be obtained from the circuit component values as follows:

$$
\begin{aligned}
& k^{2}=V_{k}=\frac{R}{R_{k}}, \quad 2 L=\frac{1}{10^{4} R_{L 1} C_{e_{1}}} \text { and } \\
& L^{2}=\frac{1}{10^{4} R_{L 2} C_{\eta}} .
\end{aligned}
$$

The circuit diagrams of the drive system, response system and feedback coupling are presented in Figs. 11, 12 and 13 , respectively.

On the feedback coupling circuit diagram of Fig. 13, the block 'SQRT' (which is found in the Pspice library and which can be easily designed by the analog devices AD633JN multiplier) represents the circuit that achieves the square root, while the one in red box represents the circuit that achieves the division operation. The resistor $R_{D 2}$ is used for tuning the division oper- 
ation. According to the selected synchronization time of the previous section $T_{\mathrm{M}}=0.950346 \mathrm{~s}$ for numerical simulations (for $k=1$ and $\hat{e}_{1}(0)=1.1$ ), the corresponding synchronization time for the Pspice simulations can be obtained as follows (see Eq. 9):

$$
\begin{aligned}
T_{\mathrm{S}} & =\mathrm{RCT}_{\mathrm{M}}=95.0346 \times 10^{-6} \mathrm{~s} \\
& =95.0346 \mu \mathrm{s},
\end{aligned}
$$

where $T_{\mathrm{S}}$ is the established synchronization time through Pspice simulations, $T_{\mathrm{M}}$ is the established synchronization time using numerical simulations, $R=$ $10 \mathrm{k} \Omega$, and $C=10 \mathrm{nF}$.

Assume that the initial conditions of the master system, slave system and feedback coupling were, respectively, chosen to be $\left(V_{C_{x_{1}}}(0), V_{C_{x_{2}}}(0), V_{C_{x_{3}}}(0)\right.$, $\left.V_{C_{x_{4}}}(0)\right)=(0.1,0.9,0.003,0.001),\left(V_{C_{y_{1}}}(0), V_{C_{y_{2}}}(0)\right.$, $\left.V_{C_{y_{3}}}(0), V_{C_{y_{4}}}(0)\right)=(0.01,2,0.8,0.1)$ and $\left(V_{e_{1}}(0)\right.$, $\left.V_{C_{\eta}}(0), V_{C_{D}}(0)\right)=(-1.1,0,0)$. The circuit component values of the feedback coupling were chosen to be $C_{e_{1}}=C_{\eta}=C=10 \mathrm{nF}, C_{D}=22 \mathrm{pF}, R_{D 1}=R=$ $10 \mathrm{k} \Omega, R_{D 2}=50 \mathrm{k} \Omega, R_{L 1}=25 \Omega, R_{L 2}=0.25 \Omega$, $R_{k}=10 \mathrm{k} \Omega$, and $V_{k}=1 \mathrm{~V}$. The voltage sources is set at $\pm 15 \mathrm{Vdc}$. It is noted that the component values of the master are the same with the component values of the slave: $R_{c}=8 \mathrm{k} \Omega, R_{d}=4 \mathrm{k} \Omega$ and $R_{b}=250 \Omega$. It is noted that the effects of varying parameter $k$ on the synchronization time (finite horizon) can be analyzed by monitoring the resistor $R_{k}$ and voltage $V_{k}$ while respecting the relation $k^{2}=V_{k}=\frac{R}{R_{k}}$. The Pspice simulation results of the proposed synchronization scheme are shown in Figs. 14 and 15. Note that a fairly good convergence of error $e_{2}$ is obtained in about $95.0346 \mu \mathrm{s}$ which corresponds to the finite horizon (see Fig. 14). One can observe the good correspondence with numerical simulations of the previous section (see Fig. 8). Then, the Pspice results confirm the practical applicability of the proposed method.

\section{Conclusion}

In this paper, a four-dimensional autonomous chaotic system obtained by the modification of a twodimensional Rayleigh system is presented. The chaotic behavior of the model was analyzed numerically. The largest 1D numerical Lyapunov exponent and the bifurcation diagram were used as indicators of chaotic motion. An appropriate analog simulator was designed. The results from the electronic circuit were compared to the numerical results, and a very good agreement between the two methods was found. Furthermore, the synchronization of such proposed systems was also addressed; a novel robust control scheme using the controllability functions method for the synchronization between two modified Rayleigh systems is presented. The main idea is to construct an augmented dynamical system from the synchronization error system, which is itself uncertain. Then, we have proposed a robust feedback coupling that takes into account the behavior of transient response and the feedback coupling effort (i.e., the energy wasted by the feedback coupling action). Thus, the proposed strategy allows to set specifically the time horizon for the synchronization of two modified systems. Both stability analysis and numerical simulations are presented to show the effectiveness of the optimization strategy. Also, Pspice simulations are presented to show the feasibility of the proposed scheme.

Acknowledgments The authors would like to thank the Nuclear Instrumentation Unit of the Nuclear Technology Section, Institute of Geological and Mining Research, for providing electronic equipments and facilities that helped to achieve the experimental part of the work. We also thank the anonymous reviewers for their helpful and constructive comments that greatly contributed to improving the final version of the paper, as well as the editors for their generous comments and support during the review process. Patrick Louodop acknowledges the support by Grant Nr. 2014/13272-1 Sao Paulo Research Foundation (FAPESP), Brazil.

\section{References}

1. Lorenz, E.N.: Deterministic non periodic flow. J. Atmos. Sci. 20, 130-141 (1963)

2. Rössler, O.E.: An equation for continuous. Phys. Lett. 57A, 397-398 (1976)

3. Rössler, O.E.: An equation for hyper chaos. Phys. Lett. A 71, 155-157 (1979)

4. Polianshenko, M., McKay, S.R.: Chaos due to homoclinic and heteroclinic orbits in two coupled oscillators with nonisochronism. Phys. Rev. A 46, 5271-5274 (1992)

5. Chen, G., Dong, X.: From Chaos to Order: Methodologies, Perspectives and Applications. World Scientific, Singapore (1998)

6. Goedgebuer, J.P., larger, L., Porle, H.: Optical cryptosystem based on synchronization of hyper chaos generated by a delayed feedback tunable laser diode. Phys. Rev. Lett. 80, 2249-2252 (1998)

7. Kozowski, T.J., Parlitz, U., Lauterborn, W.: Bifurcation analysis of two coupled periodically driven Duffing oscillators. Phys. Rev. E 51, 1861-1867 (1995)

8. Lord Rayleigh (J. W. Strutt): On maintained vibrations. Philos. Mag. XV, 229-232 (1883) 
9. Khalil, H.: Nonlinera Systems. Prentice Hall, Englewood Cliffs, NJ (2002)

10. Diener, M.: Nessie et les canards. IRMA, Strasbourg (1979)

11. Diener, M.: Quelques Examples de bifurcations et les canards. IRMA, Strasbourg, 1979-1985 (1979)

12. Southerland, K.B., Frederiksen, R.D., Dahm, W.J.A., Dowling, D.R.: Comparisons of mixing in chaotic and turbulent flows. Chaos Solitons Fractals 4, 1057-1089 (1994)

13. Cuomo, K.M., Oppenheim, A.V.: Circuit implementation of synchronized chaos with applications to communications. Phys. Rev. Lett. 71, 65-68 (1993)

14. Femat, R., Jauregui-Ortiz, R., Solis-Perales, G.: A chaosbased communication scheme via robust asymptotic feedback. IEEE Trans. Circuits Syst. I 48, 1161-1169 (2001)

15. Wang, X.F., Chen, G.: Chaotifying a stable map via smooth small-amplitude high-frequency feedback control. Int. J. Circuit Theory Appl. 28, 305-312 (2000)

16. Bowong, S.: Tracking control of nonlinear chaotic systems with dynamics uncertainties. J. Math. Anal. Appl. 328, 842859 (2007)

17. Bollt, E., Kostelich, E.: Optimal targeting of chaos. Phys. Lett. A 245, 399-406 (1998)

18. Femat, R., Alvarez-Ramirez, J., Gonzales, J.A.: A strategy to control chaos in nonlinear driven oscillators with least prior knowledge. Phys. Lett. A 224, 271-276 (1997)

19. Yassen, M.T.: Adaptive control and synchronization of a modified Chua's circuit system. Appl. Math. Comput. 135, 113-128 (2003)

20. Ahlborn, A., Parlitz, U.: Stabilizing unstable steady states using multiple delay feedback control. Phys. Rev. Lett. 93, 264101 (2004)

21. Femat, R., Alvarez-Ramirez, J.: Synchronization of a class of strictly different chaotic oscillators. Phys. Lett. A 236, 307-313 (1997)

22. Hwang, C.C., Chow, H.-Y., Wang, Y.-K.: A new feedback control of a modified Chua's circuit system. Phys. D 92, 95-100 (1996)

23. Fotsin, H., Bowong, S., Daafouz, J.: Adaptive synchronization of two chaotic systems consisting of modified Van der Pol-Duffing and Chua oscillators. Chaos Solitons Fractals 26, 215-29 (2005)

24. Bowong, S., Moukam Kakmeni, F.M., Fotsin, H.: A new adaptive observer-based synchronization scheme for private communication. Phys. Lett. A 355, 193-201 (2006)

25. Solis-Perales, G., Femat, R., Ruiz-Velasquez, E.: A note on robust stability analysis of chaos synchronization. Phys. Lett. A 288, 183-190 (2001)

26. Louodop, P., Fotsin, H., Bowong, S., Kammogne, T.S.A.: Adaptive time-delay synchronization of chaotic systems with uncertainties using a nonlinear feedback coupling. J. Vib. Control 20(6), 815-826 (2014)

27. Louodop, P., Fotsin, H., Kountchou, M., Ngouonkadi, L.B.M., Cerdeira, H.A., Bowong, S.: Finite-time synchronization of tunnel-diode-based chaotic oscillators. Phys. Rev. E 89, 032921 (2014)

28. Fotsin, H.B., Kakmeni, F.M., Bowong, S.: An adaptive observer for chaos synchronization of a nonlinear electronic circuit. Int. J. Bifurcat. Chaos 16, 2671-2679 (2006)

29. Fotsin, H.B., Bowong, S.: Adaptive control and synchronization of chaotic systems consisting of Van der Pol oscil- lators coupled to linear oscillators. Chaos Solitons Fractals 27, 822-835 (2006)

30. Fotsin, H.B., Daafouz, J.: Adaptive synchronization of uncertain chaotic colpitts oscillators based on parameter identification. Phys. Lett. A 339, 304-315 (2005)

31. Itoh, M., Yang, T., Chua, L.O.: Conditions for impulsive synchronization of chaotic and hyperchaotic systems. Int. J. Bifurcat. Chaos 11, 551-560 (2001)

32. Bowong, S., Moukam Kakmeni, F.M.: Synchronization of uncertain chaotic systems via backstepping approach. Chaos Solitons Fractals 21, 999-1011 (2004)

33. Lü, J., Zhang, S.: Controlling Chen's chaotic attractor using backstepping design based on parameters identification. Phys. Lett. A 286, 148-152 (2001)

34. Wang, C., Ge, S.S.: Synchronization of two uncertain chaotic systems via adaptive backstepping. Int. J. Bifurcat. Chaos 11, 1743-1751 (2001)

35. Femat, R., Solis-Perales, G.: Synchronization of chaotic systems with different order. Phys. Rev. E 65, 036226-0362233 (2002)

36. Chedjou, J.C., Fotsin, H.B., Woafo, P., Domngang, S.: Ana$\log$ simulation of the dynamics of a van der Pol oscillator coupled to a Duffing oscillator. IEEE Trans. Circuits Syst. I Fundam. Theory Appl. 48, 748-756 (2001)

37. Kengne, J., Chedjou, J.C., Kenne, G., Kyamakya, K., Kom, G.H.: Analog circuit implementation and synchronization of a system consisting of a van der Pol oscillator linearly coupled to a Duffing oscillator. Nonlinear Dyn. 70, 2163 2173 (2012)

38. Johnson, C.I.: Analog Computer Techniques. Mc-GrawHill, New York (1963)

39. Sheingold, D.H.: Nonlinear Circuits Handbook. Analog Devices, Norwood, MA (1976)

40. Parker, T.S., Chua, L.O.: Chaos: a tutorial for engineers. Proc. IEEE 75, 982-1008 (1987)

41. Hamill, D.C.: Learning about chaotic circuits with SPICE. IEEE Trans. Educ. 36, 28-35 (1993)

42. Louodop, P., Fotsin, H., Kountchou, M., Bowong, S.: Finitetime synchronization of Lorenz chaotic systems: theory and circuits. Phys. Scr. 88, 045002 (2013)

43. Kountchou, M., Louodop, P., Bowong, S., Fotsin, H.: Optimization of the synchronization of the modified Duffing system. J. Adv. Res. Dyn. Control Syst. 6, 25-48 (2014)

44. Korobov, V.I., Krutin, V.I., Sklyar, G.M.: An optimal control problem with a mixed cost function. Siam J. Control Optim. 31, 624-645 (1993)

45. Isidori, A.: Non-linear Control Systems, 2nd edn. Springer, Berlin (1989)

46. Kocarev, L., Parlitz, U., Hu, B.: Lie derivatives and dynamical systems. Chaos Solitons Fractals 9, 1359-1366 (1998)

47. Femat, R., Jiménez, C., Bowong, S., Solís-Perales, G.: Accounting the control effort to improve chaos suppression via robust adaptive feedback. Int. J. Model. Identif. Control 6, 147-155 (2009)

48. Femat, R., Alvarez-Ramírez, J., Castillo-Toledo, B., Gonzáles, J.: On robust chaos suppression in a class of non driven oscillators: application to the Chua's circuit. IEEE Trans. Circuits Syst. I(46), 1150-1162 (1999)

49. Femat, R., Solís-Perales, G.: Robust Synchronization of Chaotic Systems via Feedback. Springer, Berlin (2008) 